\title{
Epidemiologia da leishmaniose visceral humana em Petrolina, Pernambuco, no período de 2009 a 2020: uma análise descritiva
}

\author{
Epidemiology of human visceral leishmaniasis in Petrolina, Pernambuco, from 2009 to 2020: a \\ descriptive analysis \\ Epidemiología de leishmaniasis visceral humana en Petrolina, Pernambuco, de 2009 a 2020: un \\ análisis descriptivo
}

\author{
Márcia Marília de Souza Silva \\ ORCID: https://orcid.org/0000-0002-0159-8759 \\ Universidade de Pernambuco, Brasil \\ E-mail: marciamarilia75@gmail.com \\ Julia Millena dos Santos Silva \\ ORCID: https://orcid.org/0000-0003-4912-1076 \\ Universidade de Pernambuco, Brasil \\ E-mail: juliasilva1js@gmail.com \\ Danielle de Sousa Alves \\ ORCID: https://orcid.org/0000-0002-6431-053X \\ Universidade de Pernambuco, Brasil \\ E-mail: daniellesous@hotmail.com \\ Michele Vantini Checchio Skrapec \\ ORCID: https://orcid.org/0000-0001-5681-5987 \\ Universidade de Pernambuco, Brasil \\ E-mail: michele.skrapec@upe.br \\ Diego Barbosa de Queiroz \\ ORCID: https://orcid.org/0000-0003-3105-1428 \\ Universidade de Pernambuco, Brasil \\ E-mail: diego.queiroz@upe.br \\ Diego Felipe dos Santos Silva \\ ORCID: https://orcid.org/0000-0003-3105-1428 \\ Universidade de Pernambuco, Brasil \\ E-mail: diego.santos@upe.br \\ Marcos Veríssimo de Oliveira Cardoso \\ ORCID: https://orcid.org/0000-0001-8676-1115 \\ Universidade de Pernambuco, Brasil \\ E-mail: marcos.cardoso@upe.br
}

\begin{abstract}
Resumo
Introdução: A Leishmaniose visceral (LV) é classificada como uma doença tropical negligenciada, causada por espécies do gênero Leishmania que tem como vetor inseto flebótomo do gênero Lutzomyia. A região nordeste do Brasil ainda apresenta o maior número de casos, bem como o município de Petrolina, localizado no interior do estado de Pernambuco, que registra anualmente novos casos. Assim, o objetivo do trabalho foi descrever as características epidemiológicas dos casos de leishmaniose visceral em Petrolina no período de 2009 a 2020. Métodos: O levantamento foi realizado a partir de informações disponíveis na base de dados do Sistema de Informações de Agravos de Notificações e na Secretaria de Saúde do município de Petrolina. Foram calculados indicadores epidemiológicos e, para isso, foram utilizados dados referentes ao sexo, faixa-etária, raça, zona de residência, evolução do caso, critério de confirmação e coinfecção com HIV. Resultados: No período estudado foram confirmados 354 casos com coeficiente de incidência superior à média nacional, além disso, a doença foi observada predominantemente no sexo masculino, na raça parda, em indivíduos que residem na zona urbana da cidade e durante a infância. Conclusões: Os dados disponibilizados nos bancos de dados ainda possuem falhas, a falta de informações completas nas fichas de notificações leva a falha na investigação da doença e, consequentemente, uma incidência subestimada.
\end{abstract}

Palavras-chave: Leishmaniose; Petrolina; Epidemiologia.

\begin{abstract}
Introduction: Visceral Leishmaniasis (VL) is classified as a neglected tropical disease caused by the genus Leishmania, which has as vector the sand fly insect of the genus Lutzomyia. The northeast region of Brazil still has
\end{abstract}


the highest number of cases, and the municipality of Petrolina, located in the inland of the state of Pernambuco, registers all new cases. Thus, the objective of the study described the epidemiological characteristics of cases of visceral leishmaniasis in Petrolina from 2009 to 2020. Methods: The survey was carried out based on information available in the available database of the Information System for Diseases of Notification and in the Health Department of the municipality of Petrolina. Epidemiological indicators were calculated and, for this, data referring to sex, age group, race, area of residence, case evolution, confirmation criteria, and HIV co-infection were used. Results: In the period studied, 354 cases were confirmed with a treatment coefficient higher than the national average. In addition, the disease was observed predominantly in males, brown race, in non-residents in the urban area of the city, and during childhood. Conclusions: The data available in the databases still have flaws. A lack of complete information in the notification forms leads to failure in investigating the disease and, consequently, an underestimated incidence. Thus, the results obtained contribute to a better understanding of the current scenario of leishmaniasis in the city of Petrolina-PE.

Keywords: Leishmaniasis; Petrolina; Epidemiology.

\section{Resumen}

Introducción: La leishmaniasis visceral (LV) se clasifica como una enfermedad tropical desatendida, causada por especies del género Leishmania que tiene como vector al insecto mosca de la arena del género Lutzomyia. La región noreste de Brasil todavía tiene el mayor número de casos, así como el municipio de Petrolina, ubicado en el interior del estado de Pernambuco, que anualmente registra nuevos casos. Así, el objetivo del estudio fue describir las características epidemiológicas de los casos de leishmaniasis visceral en Petrolina de 2009 a 2020. Métodos: La encuesta se realizó con base en la información disponible en la base de datos del Sistema de Información de Enfermedades de Notificación y en el Departamento de Salud del municipio de Petrolina. Se calcularon indicadores epidemiológicos y, para ello, se utilizaron datos referentes al sexo, grupo de edad, raza, área de residencia, evolución del caso, criterios de confirmación y coinfección por VIH. Resultados: Durante el período de estudio se confirmaron 354 casos con una tasa de incidencia superior a la media nacional, además, la enfermedad se observó predominantemente en varones, raza morena, en individuos residentes en el área urbana de la ciudad y durante la niñez. Conclusiones: Los datos disponibles en las bases de datos aún presentan fallas, la falta de información completa en los formularios de notificación conduce al fracaso en la investigación de la enfermedad y, en consecuencia, a una incidencia subestimada.

Palabras clave: Leishmaniasis; Petrolina; Epidemiología.

\section{Introdução}

A Leishmaniose visceral (LV) é classificada como uma doença tropical negligenciada não contagiosa e de transmissão vetorial, reconhecida pela Organização Mundial de Saúde (OMS) como uma das infecções parasitárias mais comuns no mundo, ainda continua em crescimento no Brasil (Benchimol et al., 2019; Meira \& Gedamu, 2019).

A LV é causada por espécies do gênero Leishmania, parasitas intracelulares obrigatórios com ciclo de vida digenético, entre o vetor flebótomo do gênero Lutzomyia e um mamífero. No Brasil é causada principalmente pela espécie Leishmania infantum transmitida pelo inseto Lutzomyia longipalpis, conhecido popularmente como mosquito palha, asa-dura, tatuquiras, birigui, dentre outros (Ministério da Saúde, 2019).

Flebótomos fêmea, quando infectadas, transmitem formas promastigotas do parasita durante a sua alimentação com sangue de mamíferos, ao entrarem na corrente sanguínea essas formas são fagocitadas por células do sistema mononuclear fagocitário, onde se diferenciam em amastigotas, se proliferam e estabelecem a infecção em fagossomos até o seu rompimento e liberação dos parasitas, que serão novamente parasitados em um ciclo contínuo (Meira \& Gedamu, 2019). No novo mundo, em especial no Brasil, os principais reservatórios da doença em vertebrados são cães, raposas, outros mamíferos e pessoas infectadas (Costa, 2011).

Pacientes com LV podem desenvolvê-la de forma assintomática ou apresentarem manifestações clínicas que envolvem febre persistente, perda de peso e anemia, que podem evoluir para esplenomegalia e hepatomegalia (Meira \& Gedamu, 2019).

A LV é descrita como endêmica em pelo menos 76 países no mundo e em 12 do continente americano, sendo que, destes, grande parte ocorre no Brasil, com uma média de 3.500 casos registrados ao ano. O primeiro caso da doença no território nacional foi descrito em 1913 no município de Boa Esperança, Mato Grosso, e desde então vem sendo descrita em 
várias regiões do país (Ministério da Saúde, 2019). O controle da doença teve início no estado do Ceará em 1959 seguindo três estratégias: o tratamento dos pacientes, o uso de DDT (diclorodifeniltricloroetano) e a eliminação dos cães com sorologia reativa (Costa, 2011).

O Nordeste do Brasil apresenta o maior número de casos confirmados. Pernambuco registrou 1465 casos, no período de 2000 a 2010, nos quais mais de 100 casos ocorreram em Petrolina, localizada no sertão do estado. Fatores como urbanização, condições precárias de vida, falta de saneamento básico e destinação inadequada de resíduos sólidos podem ter contribuído para a expansão e incidência da doença na cidade (Maia et al., 2013).

Considerando que os estudos epidemiológicos trazem contribuições relevantes para as atividades de informação sanitária, controle e prevenção da leishmaniose junto à comunidade e tomada de decisões por parte dos gestores regionais, o objetivo do presente estudo foi descrever as características epidemiológicas dos casos de leishmaniose visceral - LV notificados no município de Petrolina, estado de Pernambuco, Brasil, no período de 2009 a 2020.

\section{Metodologia}

\subsection{Delineamento da pesquisa}

Trata-se de um estudo descritivo, longitudinal e retrospectivo, com abordagem quantitativa dos casos de Leishmaniose Visceral Humana (LV) no município de Petrolina, entre os anos de 2009 e 2020.

\subsection{Coleta de dados}

O levantamento foi realizado a partir de informações disponíveis na base de dados do Sistema de Informações de Agravos de Notificações (SINAN) e na Secretaria de Saúde do município de Petrolina-PE, no período de dezembro de 2020 a fevereiro de 2021.

Foram calculados indicadores epidemiológicos destinados à avaliação da conjuntura da LV no município e, para isso, foram utilizados dados referentes ao sexo, faixa-etária, raça, zona de residência, evolução do caso, critério de confirmação e coinfecção com HIV.

\section{3 Ética}

Este trabalho não requer autorização do Comitê de Ética em Pesquisa por não utilizar material biológico nem variáveis que possibilitaram a identificação dos indivíduos cujos dados foram objetos da pesquisa, de acordo com a Resolução do Conselho Nacional de Saúde (CNS) nº 466, 12 de dezembro de 2012.

\section{Resultados e Discussão}

A doença ocorre em todo o território nacional e no período de 2009 a 2019 foram registrados 40.326 casos, com uma média de 3.666 casos ao ano. Neste mesmo período, Pernambuco registrou 1.340 casos, em média 121 casos por ano, tendo um aumento considerável a partir de 2014. Essa parasitose já foi considerada controlada no Brasil, entretanto, vem apresentando aumento no número de ocorrências, além de uma vasta expansão geográfica, adquirindo formas graves que, quando associadas à má nutrição e coinfecção com outras doenças, torna-se letal (Benchimol et al., 2019).

A LV foi confirmada em 76 municípios pernambucanos, destes, a capital Recife registrou o maior número de ocorrências, com 30,82 \% dos casos confirmados, seguido por Petrolina com 14,1 \% e Salgueiro com 10,74 \%, localizados no sertão do estado. O número de casos registrados nesses três municípios representa mais da metade do total de casos confirmados em todo o estado, se fazendo necessário o melhor entendimento sobre o perfil epidemiológico dessas regiões.

O município de Petrolina é considerado uma importante área endêmica na região. Um estudo anterior feito por Maia 
et al. (Maia et al., 2013) (5) revelou fatores de risco que contribuem para a manutenção e expansão da doença em Petrolina, no qual o comportamento das práticas cotidianas é o principal fator, mesmo entre a população que conhece o papel do cão e do vetor na doença.

No período de 2009 a 2020, foram confirmados 354 casos de LV em Petrolina (Brasil, 2021) e, a partir destes, foi calculado o coeficiente de incidência da doença, demonstrando a intensidade com a qual a morbidade incidiu sobre a população nesse intervalo de tempo (Tabela 1).

Tabela 1. Coeficiente de incidência da leishmaniose visceral no município de Petrolina de 2009 a 2020.

\begin{tabular}{lccc}
\hline Ano & Casos confirmados & População estimada & Coeficiente por 100 mil hab. \\
\hline $\mathbf{2 0 0 9}$ & 10 & 293.930 & 3,4 \\
\hline $\mathbf{2 0 1 0}$ & 17 & 300.794 & 4,65 \\
\hline $\mathbf{2 0 1 1}$ & 14 & 306.123 & 4,57 \\
\hline $\mathbf{2 0 1 2}$ & 32 & 311.700 & 10,26 \\
\hline $\mathbf{2 0 1 3}$ & 38 & 317.178 & 11,98 \\
\hline $\mathbf{2 0 1 4}$ & 39 & 322.645 & 12,08 \\
\hline $\mathbf{2 0 1 5}$ & 34 & 328.317 & 10,35 \\
\hline $\mathbf{2 0 1 6}$ & 34 & 333.497 & 10,19 \\
\hline $\mathbf{2 0 1 7}$ & 38 & 338.488 & 11,22 \\
\hline $\mathbf{2 0 1 8}$ & 40 & 343.865 & 11,63 \\
\hline $\mathbf{2 0 1 9}$ & 32 & 349.145 & 9,16 \\
\hline $\mathbf{2 0 2 0}$ & 26 & 354.317 & 7,33 \\
\hline
\end{tabular}

Fonte: SINAN (2021).

O ano de 2009 apresentou o menor coeficiente de incidência para o período estudado (3,4/100 mil hab.), ainda assim esse valor é superior à média nacional que é de 2,0 casos para cada 100 mil habitantes (Ministério da Saúde, 2019). Foi possível observar que nos anos 2012 a 2018 houve uma persistência e intensidade da doença na população, com valores se mantendo acima de 10 casos por 100 mil habitantes. $\mathrm{O}$ ano de 2014 foi o que apresentou o maior valor (12,08/100 mil hab.), sendo superior ao coeficiente de incidência nacional para o mesmo período, que correspondeu a 1,84/100 mil habitantes.

Quanto as características analisadas, Petrolina mostrou que a maioria dos pacientes eram do sexo masculino (61 \%), este resultado reforça estudos anteriores que indicam que os homens representam o maior percentual de infectados devido a relação com as suas atividades ocupacionais (Araújo \& Nunes, 2017; Brazuna et al., 2012). A faixa-etária variou entre zero até mais de 80 anos, com as maiores ocorrências acontecendo ainda na infância até os 9 anos (48,6 \%). Esses resultados corroboram com a revisão feita por Dantas-Torres (Dantas-Torres, 2006) e consolidam informações disponibilizadas por Brasil (Ministério da Saúde, 2014), as quais indicam que a maior vulnerabilidade das crianças se explica pela imaturidade imunológica agravada pela desnutrição. Os pacientes com idade entre 20 e 64 anos também demonstraram um valor considerável (39,3\%), esses resultados também foram observados em outro estudo (Araújo \& Nunes, 2017) os quais identificam que essa faixa-etária corresponde à população economicamente ativa que estão mais expostos aos vetores durante as suas atividades cotidianas.

A maioria dos casos confirmados foram de pacientes da raça parda (253), representando $71 \%$ dos casos, entretanto, vale ressaltar que grande parte da população petrolinense se autodeclara parda, o que justifica a elevada quantidade de casos para essa raça (IBGE, 2010). Em seguida vem a raça identificada como ignorado/branco (46), sendo esse um dado bastante relevante para traçar o perfil epidemiológico, no entanto, muitas vezes é ignorado. As raças indígena e amarela apresentaram 
os menores valores, com 1 e 2 casos, respectivamente.

A maioria dos casos de LV foi descrita como sendo da área urbana (71\%), seguido por rural (25\%). Esses dados corroboram com um estudo de Maia et al. (Maia et al., 2013), apresentando uma tendência de urbanização da doença. As modificações ambientais provocadas pelo fluxo migratório, principalmente por causas socioeconômicas, levam a uma rápida e desorganizada urbanização, associadas a quintais com potencial para reprodução de flebotomíneos e a presença de animais domésticos nas residências, ocasionam um aumento das áreas endêmicas, bem como o aparecimento de novos focos da doença (Marcondes \& Rossi, 2013; Ministério da Saúde, 2014).

Apesar de haver tratamento para a leishmaniose este ainda é baseado em poucas quimioterapias e envolve efeitos colaterais tóxicos, com eficácia variável e resistência ao medicamento, fazendo com que os pacientes abandonem o tratamento ou ainda que este não seja eficaz, dependendo do grau de evolução da doença (Meira \& Gedamu, 2019). Quando não tratada, a doença pode causar, além dos sinais e sintomas já citados, infecções bacterianas, anemia grave, sangramento ou insuficiência hepática (Wilhelm, 2019). A Figura 1 mostra o número de casos confirmados em Petrolina ao longo dos anos, em que grande parte dos casos apresentou sucesso na cura, entretanto ainda acontecem óbitos por esta causa, com 4 óbitos registrados e coeficiente de letalidade $1,12 \%$, visto que é uma doença mundialmente conhecida, porém pouco se avança em novas terapias ou intervenções para erradicar a doença. No Brasil, a taxa de letalidade vem aumentando ao longo dos anos, passando de 3,1\% em 2000 para 7,1 \% em 2018 (Ministério da Saúde, 2019).

Figura 1. Número de casos confirmados segundo a evolução do paciente com leishmaniose visceral no Município de Petrolina de 2009 a 2020.



Fonte: SINAN (2021).

Durante o período estudado, Petrolina não registrou o critério de confirmação para LV em $71 \%$ (250) dos casos. Os demais foram confirmados com exames laboratoriais (26\%) e avaliação clínica e/ou epidemiológica (17 \%). O diagnóstico preciso da LV ainda enfrenta problemas, mesmo quando o paciente apresenta um quadro clínico sugestivo pode haver falhas no diagnóstico, levando ao subdiagnóstico dos casos. Esse resultado pode ser intensificado quando se leva em consideração os casos assintomáticos ou de acometidos que morrem sem ter o diagnóstico da doença (Hirata et al., 2019).

Um estudo feito por Hirata (Hirata et al., 2019), usando o ensaio de imunoabsorção enzimática (ELISA), mostrou que em uma área endêmica para leishmaniose visceral, cerca de $20 \%$ dos pacientes apresentaram anticorpos anti-Leishmania spp., 
entretanto, nenhum deles tinha a LV como possível diagnóstico, revelando casos subdiagnosticados.

Os pacientes com coinfecção com HIV representaram $12 \%$ (42) dos casos. A maioria dos pacientes (56 \%) não apresentou coinfecção com HIV, entretanto, o número de pacientes que não reportaram se houve ou não coinfecção chega a 32 $\%$ (113), o que pode mascarar o resultado, como mostra a Figura 2.

Figura 2. Valores absolutos quanto a co-infecção HIV-LV em pacientes no Município de Petrolina de 2009 a 2020.

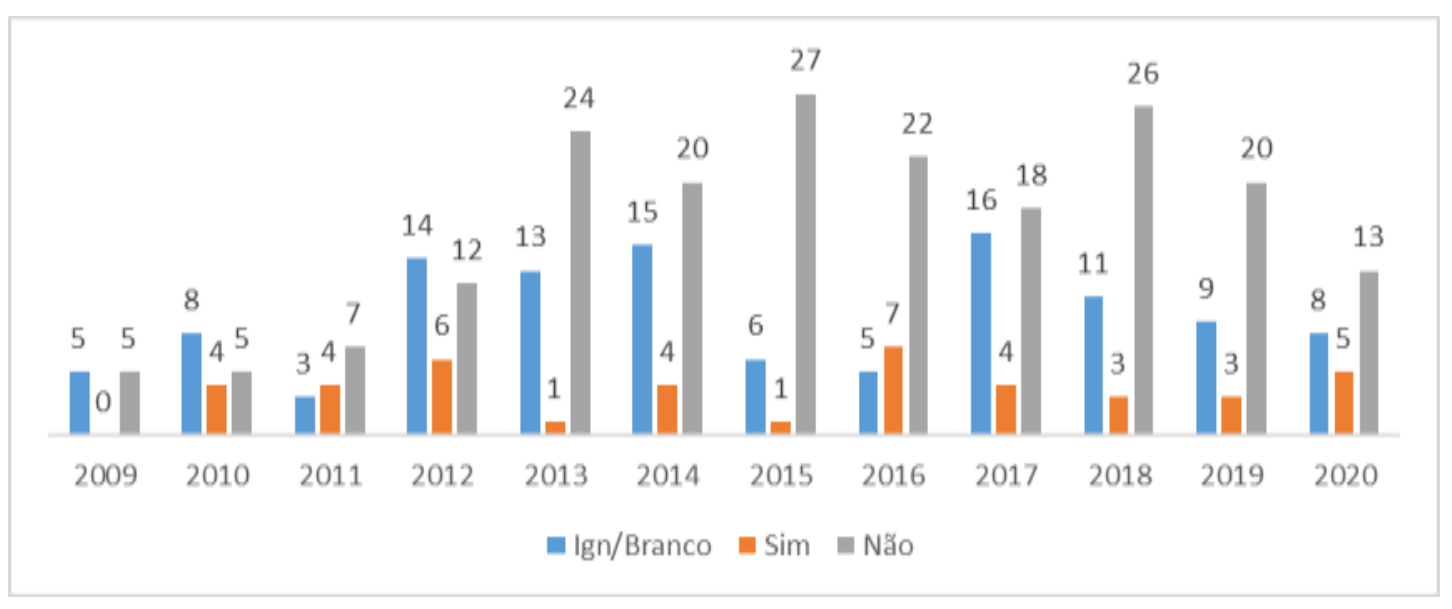

Fonte: SINAN (2021).

Em 2009 não foi registrada coinfecção com HIV, no entanto, metade dos casos registrados estavam como ignorado/branco. Os anos com o maior número de coinfectados foram 2012 e 2016, com 6 e 7 casos, respectivamente (Figura 2).

Um estudo realizado mostrou que a coinfecção por HIV-LV pode se mostrar de diferentes formas, sendo assintomática, o que dificulta o diagnóstico ou pode evoluir para formas graves com características inespecíficas (Cavalcanti et al., 2012). Os mesmos autores ainda descrevem que a LV vem sendo considerada uma infecção oportunista em pacientes com HIV e isso vem aumentando ao longo dos anos devido a ruralização do HIV e urbanização da LV.

\section{Conclusão}

O município de Petrolina apresenta um elevado número de casos de LV, sendo a cidade com o maior número de registros do interior de Pernambuco. Os resultados demonstraram que a doença se apresenta predominantemente no sexo masculino, na raça parda, em indivíduos que residem na zona urbana da cidade e durante a infância.

O sucesso no tratamento da doença não deve tirar o foco do número de pacientes que ainda morrem por esta causa, deve-se investir em estratégias não só no tratamento como também na prevenção e controle, reduzindo o número de reservatórios da doença. O elevado número de resultados como ignorado/branco em relação a coinfecção com HIV merece ser mais bem estudado.

Para traçar o perfil epidemiológico, são necessárias várias informações, entretanto, os dados disponibilizados nos bancos de dados ainda possuem falhas, a falta de informações completas nas fichas de notificações leva a falha na investigação da doença e, consequentemente, uma incidência subestimada. Desta forma, nossos resultados podem contribuir para uma melhor compreensão do atual cenário da leishmaniose no município de Petrolina-PE.

\section{Agradecimentos}

Os autores agradecem a secretaria de saúde do município de Petrolina-PE e a Fundação de Amparo à Ciência e 
Tecnologia do Estado de Pernambuco (FACEPE) pelo suporte financeiro (APQ-0350-4.03/18, PBPG-0520-4.03/19 e PBPG0358-4.03/20).

\section{Referências}

Araújo, T. T., \& Nunes, D. C. de O. (2017). A fourteen-year retrospective of clinic-epidemiological aspects of cutaneous and visceral leishmaniasis in Uberlândia, Minas Gerais, Brazil. Bioscience Journal, 33(4), 1054-1064. https://doi.org/10.14393/bj-v33n4a2017-36986.

Benchimol, J. L., Gualandi, F. da C., Barreto, D. C. dos S., \& Pinheiro, L. de A. (2019). Leishmanioses: sua configuração his tórica no Brasil com ênfase na doença visceral nos anos 1930 a 1960. Boletim do Museu Paraense Emílio Goeldi. Ciências Humanas, 14(2), 611-626. https://doi.org/10.1590/1981.81222019000200017.

Brasil. (2021). Sistema de Informação de Agravos de Notificação - Sinan. http://www2.datasus.gov.br/DATASUS/index.php?area=0203\&id=29878153.

Brazuna, J. C. M., Silva, E. A. e, Brazuna, J. M., Domingos, I. H., Chaves, N., Honer, M. R., Onselen, V. J. V., \& Oliveira, A. L. de (2012). Profile and geographic distribution of reported cases of visceral leishmaniasis in Campo Grande, State of Mato Grosso do Sul, Brazil, from 2002 to 2009. Revista da Sociedade Brasileira de Medicina Tropical, 45(5), 601-606. https://doi.org/10.1590/S0037-86822012000500012.

Cavalcanti, A. T. de A. e, Zulma, M., Fábio, L., Andrade, L. D. de, Ferreira, V. de M., Vera, M., \& Miranda-Filho, D. de B. (2012). Diagnosticando coinfecção leishmaniose visceral e HIV/AIDS: Uma série de casos em Pernambuco, Brasil. Revista Do Instituto de Medicina Tropical de Sao Paulo, 54(1), 4347. https://doi.org/10.1590/S0036-46652012000100008.

Costa, C. H. N. (2011). Quanto é efetivo o abate de cães para o controle do calazar zoonótico? Uma avaliação crítica da ciência, política e ética por trás desta política de saúde pública. Revista Da Sociedade Brasileira de Medicina Tropical, 44(2), 232-242. https://doi.org/10.1590/S0037-86822011005000014.

Dantas-Torres, F. (2006). Situação atual da epidemiologia da leishmaniose visceral em Pernambuco. Revista de Saúde Pública, 40(3), 537-541. https://doi.org/10.1590/S0034-89102006000300024.

Hirata, K. Y., Sobrinho, E. B. de O., Rigon, L., Utsunomiya, Y. T., Tomokane, T. Y., Laurenti, M. D., \& Marcondes, M. (2019). Exposure to Leishmania spp. infection and Lutzomyia spp. in individuals living in an area endemic for visceral leishmaniasis in Brazil. Revista da Sociedade Brasileira de Medicina Tropical, 53, 1-4. https://doi.org/10.1590/0037-8682-0320-2019.

IBGE. (2010). Censo 2010 Petrolina. https://cidades.ibge.gov.br/brasil/pe/petrolina/pesquisa/23/22957.

Maia, C. S., Pimentel, D. S., Santana, M. A., Oliveira, G. M., Faustino, M. A. G., \& Alves, L. C. (2013). The perception of the risk factors associated with American Visceral Leishmaniasis in Petrolina, Pernambuco, Brazil. Medicina Veterinária, 7(4), 19-25. http://ead.cod ai.ufrpe.br/index.php/medicinaveterinaria/article/view/583.

Marcondes, M., \& Rossi, C. N. (2013). Leishmaniose visceral no Brasil. Revista Brasileira de Pesquisa Veterinária e Zootecnia, 50(5), 341-352. https://doi.org/10.11606/issn.2318-3659.v50i5p341-352.

Meira, C. dos S., \& Gedamu, L. (2019). Protetor ou prejudicial? Compreendendo o papel da imunidade do hospedeiro na leishmaniose. Microorganismos, 7 (12), 695. $10.3390 /$ microorganismos7120695.

Ministério da Saúde. (2014). Leishmaniose Visceral (1ª). Secretaria de Vigilância em Saúde. Departamento de Vigilância Epidemiológica. Brasília: Ministério da Saúde.: https://bvsms.saude.gov.br/bvs/publicacoes/manual_vigilancia_controle_leishmaniose_visceral_1edicao.pdf.

Ministério da Saúde. (2019). Saúde Brasil 2018: uma análise da situação de saúde e das doenças e agravos crônicos: desafios e perspectivas (1ª). Secretaria de Vigilância em Saúde. Departamento de Vigilância de Doenças e Agravos não Transmissíveis e Promoção da Saúde. Brasília: Ministério da Saúde. https://bvsms.saude.gov.br/bvs/publicacoes/saude_brasil_2018_analise_situacao_saude_doencas_agravos_cronicos_desafios_perspectivas.pdf

Wilhelm, T. J. (2019). [Leishmaniose visceral]. Der Chirurg, 90, 833-837. https://doi.org/10.1007/s00104-019-0994-1. 